\title{
Optical Measurement Techniques for multi-dimensional measurement of Cultural Heritage
}

\author{
Stephanie Adolf \\ GOM Optical Measuring Techniques \\ Mittelweg 7-8, 38106 Braunschweig, Germany \\ s.adolf@gom.com
}

\begin{abstract}
Optical measurement systems were originally developed for industrial applications. Due to their versatility of use and "touchless" non-invasive method of working they have also been employed for many years in the field of restoration, conservation and preservation of cultural heritage. The capabilities of a variety of optical measuring methods e.g. photogrammetry, laser triangulation and interferometry have been theoretically described and their varying applications for restoration and conservation explored 10 years ago. This paper provides an overview of the utilization and implementation of Optical Measurement Systems in the field of restoration, conservation and preservation of cultural heritage that could be observed over the last ten years. Theory and function of the above mentioned systems for shape and deformation measurement of Cultural Heritage will be introduced shortly. The paper will then focus on the practical benefit of Optical Technology such as: 3D and digital archiving, monitoring of deterioration and deformation over time, precise copying and reconstruction, documentation and research, transportation packaging, etc.
\end{abstract}

Optical measurement systems, 3D laser scanning, shape \& dimension recording, monitoring deterioration and deformation, CNC-copying and reconstruction, transportation packaging

\section{INTRODUCTION}

Industrial technologies have always been investigated for wider and beneficial use in other areas. If applicable, these technologies became firmly implemented in areas such as Medicine, Entertainment or Cultural Heritage, even, in some cases, with some time delays. Of these, photogrammetry is one of the most established. This technique has been used to create true to scale drawings from images e.g. of buildings, for their digital 3D reconstruction as well as for deformation measurement. In the 1970's librarians started with the 2D digitzing of ancient books and prints for digital archiving, preservation but also to open access to a wider public via world wide web. In the 1980's curators experimented with holography to produce 3D images of objects in order to lend holographs rather than fragile originals to exhibitions. Interferometry (e.g. electronic speckle pattern interferometry) was used to detect loose paint layers, deformation of floors in historic buildings due to load as well as oscillation of musical instruments. Above all these, optical metrology systems for 3D shape and dimension measurement are today the most established in the
Cultural Heritage sector. Systems such as 3D Laserscanners or Fringe Projection Scanners seem to bring the most benefit to museums, archaeological sites as well as restoration and conservation of historic objects. The general function and common measurement workflow of these measurement systems will be described in the next chapter followed by examples of their diverse practical applications.

\section{FUNCTION AND WORKFLOW OF CLOSE RANGE SHAPE AND DIMENSION MEASUREMENT SYSTEMS}

Optical 3D measuring systems such as fringe projection systems and laser scanners have become well established in Cultural Heritage for measuring of the surface geometries of complete objects. These optical 3D coordinate measurement systems work "touchless" and are therefore non invasive, causing no harm at all to the measured object. They capture the entire component geometry in a dense point cloud instead of measuring individual points only. 
Most of these systems function on the principle of triangulation. A laser line or fringe pattern is projected on to an object and the distorted line/pattern then recorded by one or more cameras. Thus the distances for millions of points can be calculated in a few seconds, representing the geometry of the objects surface precisely.

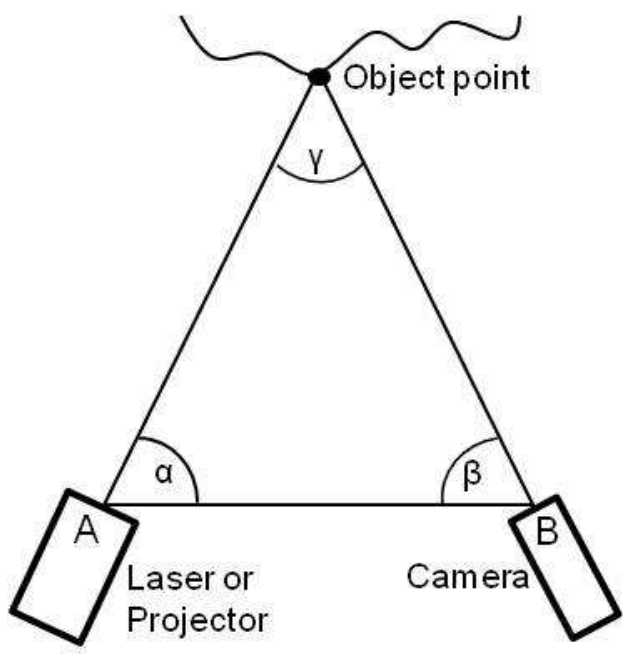

Figure 1: Principle of triangulation, if the distance between $A$ and $B$ is known and the two adjacent angles $\alpha$ and $\beta$ (due to system calibration) the distance from $B$ to the object point can be calculated.

Due to the triangulation angle the systems can only measure what they "see" which means that individual measurements need to be acquired from various directions to measure an object entirely. In most cases the user can manually position the sensor in front of the object in respect to the measuring volume. But the measuring process can also be automated by means of turntables or even robots.

Normally, a single measurement from one view takes seconds or less. There are different strategies available in order to transfer all single measurements at the right place into one common coordinate system. The transformation can be performed using reference points that were previously applied, or it can be based on geometry contours (without reference points).

In both cases the transformation should take place immediately after the measurement without user interaction and should be automatically checked by the software. The measurement system should also be self-monitoring e.g. verifying calibration status, sensor movement and ambient changes for each measurement in order to eliminate user errors and measurement artifacts during measurement process.

Once all required surfaces of the component are acquired, the individual measurements are "polygonized" in the software. Thus overlapping areas and redundant data are automatically eliminated. The result is usually a point cloud or polygon mesh also named STL Mesh (Stereolithography file format for meshed point clouds).
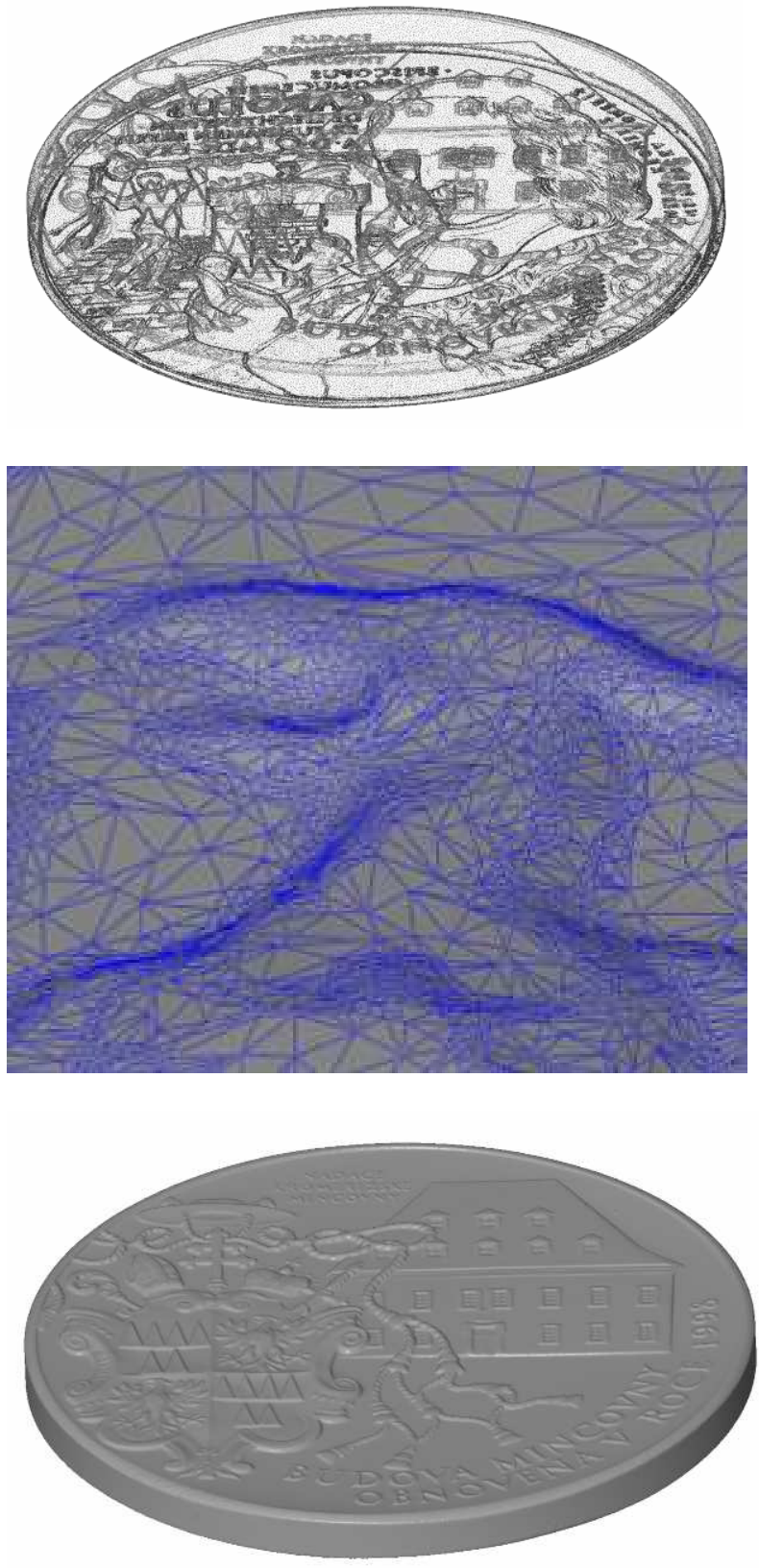

Figure 2: Point cloud, detail of meshed point cloud (STL/ polygon mesh), shaded polygon mesh (STL)

The time for data acquisition depends on the complexity of the objects shape and the size of the used measuring volume. Typically, measurement of full scale automobile by one person can be completed in less than two hours.

Most systems offer variable measuring field sizes from a few centimetres up to square meters. Thus finest details of small objects can be measured as well as components up to multiple meters in length 
with one system. The systems are usually portable and can be transported easily on-site to the object to be measured.

The data quality depends on the system used, the measuring volume and the objects surface. With measuring volumes e.g. $30 \times 30 \mathrm{~mm}$ typically accuracies of 5 microns might be achieved. For larger measuring volumes accuracies are around 15 microns. The choice of the measuring volume should depend on the smallest details of the object to be captured. Today systems with resolution between 1.4 and 8 Megapixels are common. Thus the resolution changes accordingly to the size of the measuring volume.

Dark surfaces can be measured by most systems without problems. To capture shiny surfaces more single scans might be necessary to avoid reflections. Transparent surfaces cannot be measured without preparation. Data quality is best for cooperative surfaces (dull, bright), otherwise data noise increases, but might be filtered.

\subsection{D Laser Scanners}

For scanning a surface most laser scanner use a line. Therefore the point shaped laser has to be converted to a line via a lens. To measure a surface fast the line has to be moved over the object. Some systems use a handheld approach where the user has to move the sensor constantly over the surface. Other systems use a mirror to deflect the laser line over a certain field of view.
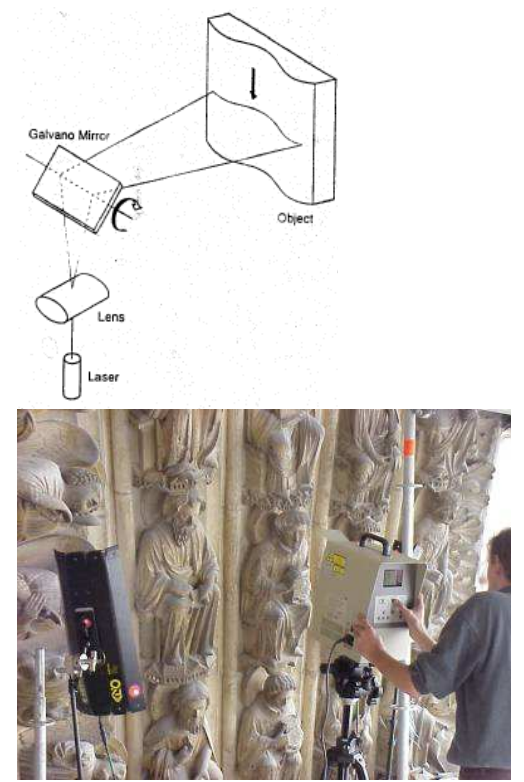

Figure 3: Measuring principle of line laser scanning system, laser scanner in use

\subsection{D Fringe Projection Scanners}

Fringe pattern projection systems usually illuminate a certain field of view which is observed by one or more cameras. The computer then calculates precise measuring points for each camera pixel in a matter of seconds. The use of stereo camera systems offers advantages compared to single camera approach. E.g. with a stereo camera setup all three viewing angles between the two cameras and projector ( $\alpha 1, \alpha 2$, and $\alpha 3$, Figure 1, left) are used. This method results in three individual viewing perspectives of the object ( 3 in 1 sensor), which greatly reduces the number of single scans.

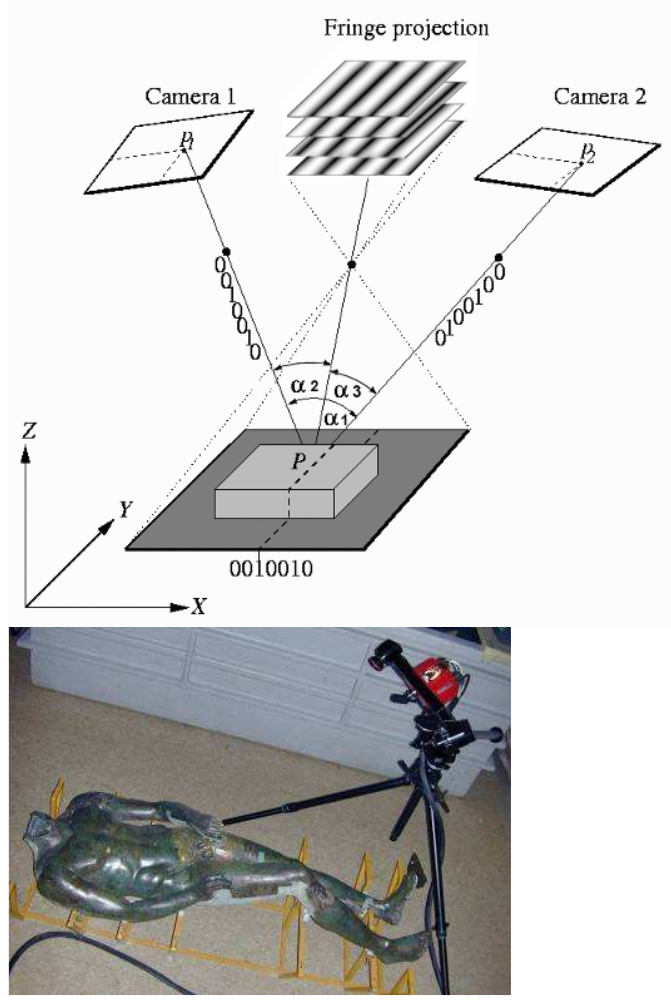

Figure 4: Measuring principle of a fringe projection system with two cameras, fringe projection system in use

\subsection{D Point Clouds / Polygon Meshes}

The geometry of objects is usually stored as point clouds or polygon meshes (ASCII, STL, etc.). In an ASCII point cloud the 3D Coordinates are sorted in three columns (XYZ) to define the object's surface. Scan data can also be processed and manipulated for easier handling. Typical functions for mesh processing are:

- Smoothing meshes

- Thinning meshes

- Hole filling in meshes

- Refinement of meshes

- Scaling to different sizes

- Extracting primitives and curvature lines

That way data can be prepared and down streamed for follow-on processes such as CNC milling, rapid prototyping, etc. 
Meshes are also suitable for analysis documentation and research. Typical functions are:

- 3D comparison of measurements

- Color deviation maps

- 2D and 3D dimensional analysis

- Inspection sections

- Cross sections

- Volume analysis

- Reports

Today there are free software packages for mesh processing and viewing up to dimensional analysis available on the internet.

\subsection{D Colour Texture Data}

Point clouds or polygon meshes can also transport colour information (PLY, OBJ, etc.). Some 3D digitizing systems use the same cameras to collect both, 3D shape and colour information within the same measurement. Other systems allow to capture geometry and texture with different systems and to precisely match colour to geometry. This way the colour information can be recorded with high-end photo cameras which are commonly used for documentation anyway and which offer a much higher resolution for texture recording than the cameras used for geometry measurement.
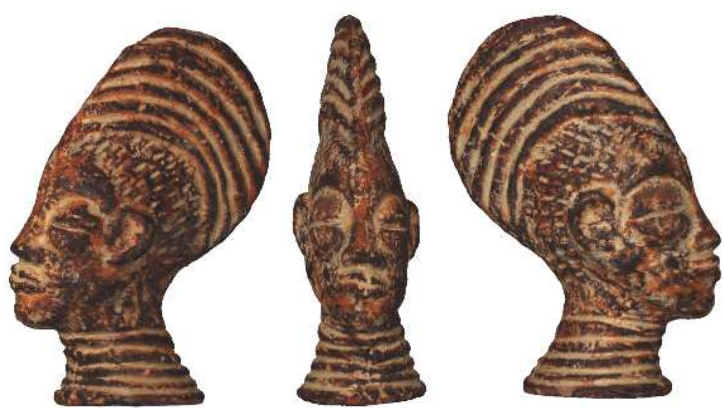

Figure 5: 3D measurement data with original colour texture documentation
3D measurement data are widely used in industrial areas as input as values for numerical simulation processes (CAE), in tool production and try-out (CAD/CAM), in inspection and quality control during production (CAQ).

Some of these processes are also most beneficial for the cultural heritage area. E.g. the dimensional analysis is used for documentation. Rapid Prototyping and CNC-Machining offer great solutions for reproduction and copying of objects without invasive moulding operations.

\subsection{D Documentation and research}

Full surface 3D data allow extensive analysis of objects of any size - non-invasive and without touching the object. The analysis of $3 \mathrm{D}$ data is a perfect tool to find out more about an object, in addition to the visual impression.

If measurement data are watertight (i.e. without holes) volume can be analysed. Any distances, angles or radii can be measured in any direction. Geometrical shapes can be extracted and the deviation to it.
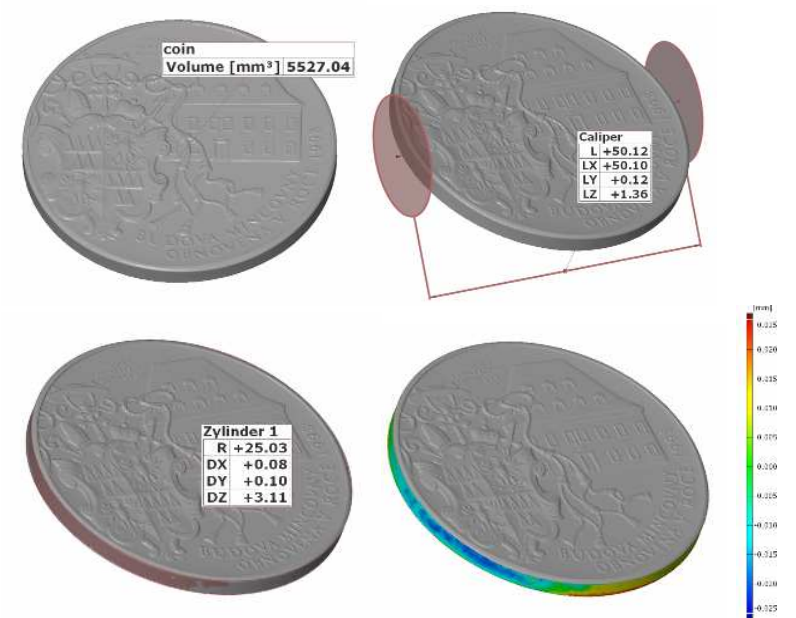

Figure 6: $3 D$ measuring data for analysis and documentation: Volume information, measurement of distances, angles, radii and analysis and comparison to geometrical shapes, e.g. circle, cylinders, etc

Detailed analysis can be also done by cross sections in any direction and distances.

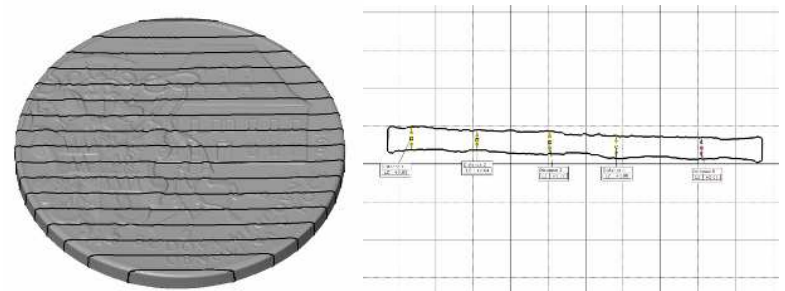

Figure 7: Cross sections with detailed analysis

3.2 Monitoring of deterioration and deformation

\section{APPPLICATIONS FOR CLOSE RANGE SHAPE AND DIMENSION MEASUREMENT SYSTEMS IN CULTURAL HERITAGE}


3D digitizing data allow also for comparison between different object stages. The deviations are coded as a full surface colour deviation plot.

Thus the Ecole d'íngénieurs de Genève in Switzerland was observing the deteoriation of an architectural detail (heads, originally painted stone), of Maison Tavel in Geneva, Switzerland, deriving from the end of the middle age.
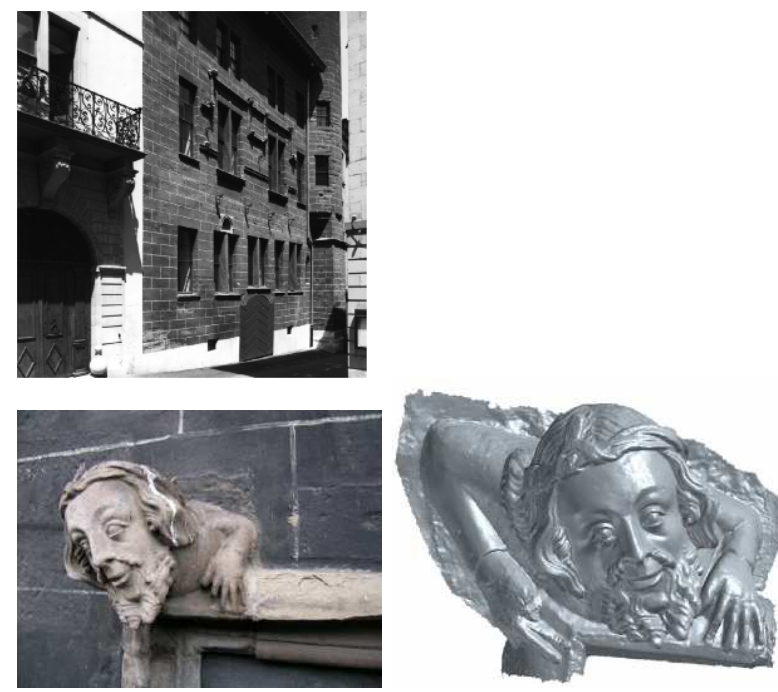

Figure 8: Maison Tavel in Geneva, Switzerland (from the end of the middle age). The heads were originally painted stone

Since 2D Images allow only a subjective estimation of damage and degradation the actual scan-data (from 2005) were compared to scan-data of plaster mouldings from 1911. Thus a qualitative and quantitative analysis of wear over 94 years was possible.

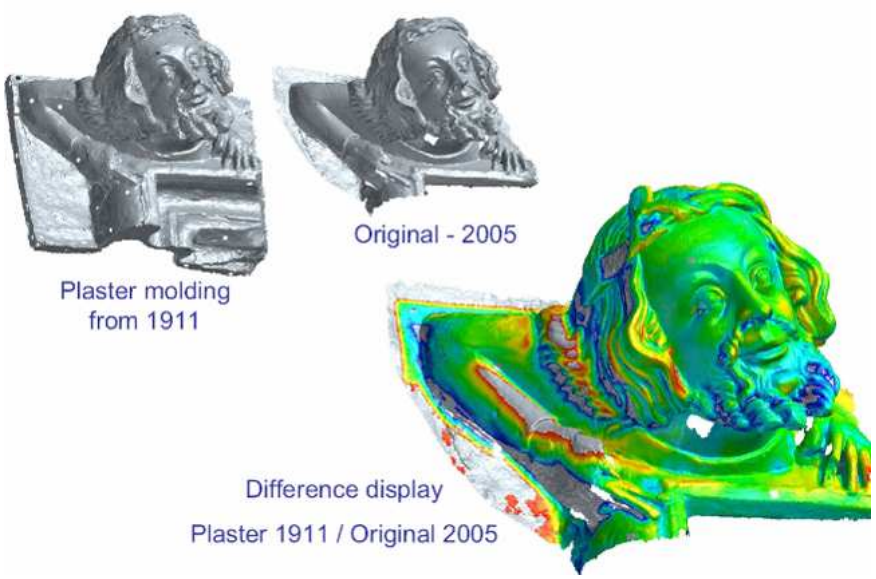

Figure 9: Comparison of scan-data from 2005 with scandata of plaster mouldings from 1911. The deviation is shown as a full field colour plot

\subsection{Copying and reconstruction}

For reconstruction, scaling and copying 3D scan data are an ideal base. The progress which CNCmilling machines and the CAD/CAM software packages have made in the last ten years is impressive. While ist was in 2000 only possible to scan small areas $(5 \times 5 \mathrm{~cm})$ and to feed these data to milling machines (Köhler 2000), only a few years later complete statues from $3 \mathrm{~m}$ height could be scanned and milled from one massive block of stone.

Thus a figure of Christ made of stone (around 1220/30) which was originally made for cloister Reichenbach (South Germany) and is now located in the Bayerisches Nationalmuseum in Munich could be scanned in the museum and a copy milled from these data in stone could be relocated into the cloister (Mühlbauer 2004).
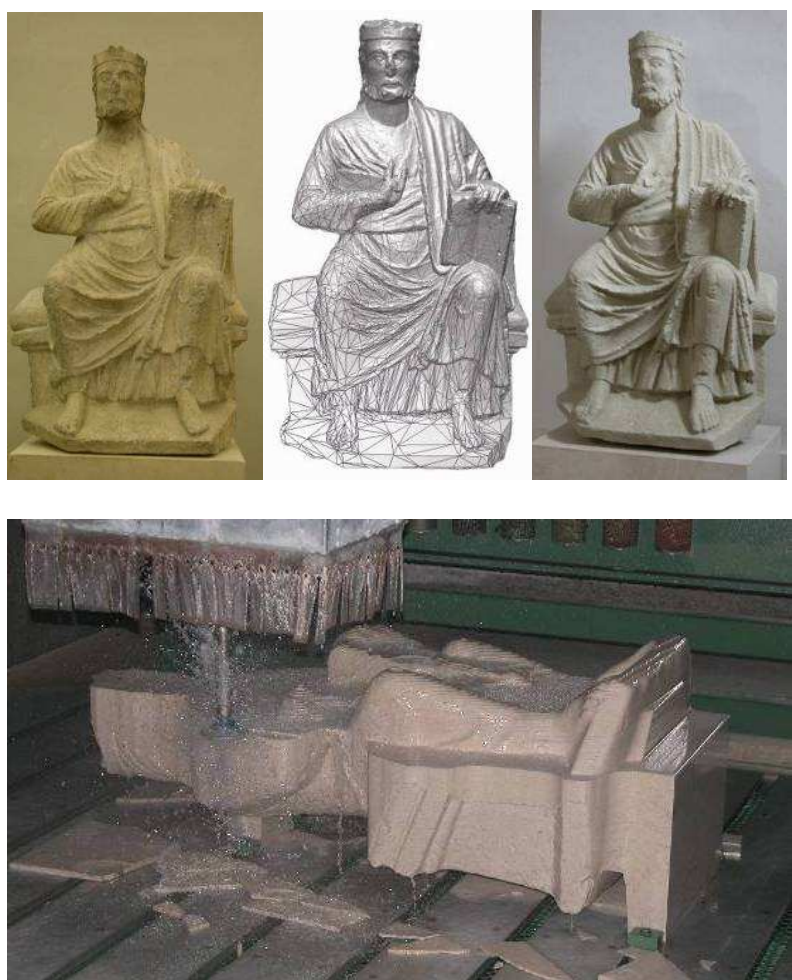

Figure 10: Figure of Christ (around 1220/30, 1,30 m height), original, 3D scan data, copy made from scan data, milling process

The utilization of 3D scanning and CNC-milling for cultural heritage results in much more precise copies than possible with the manual point wise copying by a stone mason. The process is also faster, since the rough work is done by a machine and the stone mason needs only to do fine work on the surface.

This method can also be applied to copy and scale statues. Such a project was done to complete the town hall in Graz, Austria. A model of the allegory statue "craft" had to be produced in large scale to be placed on the facade of the town hall. The small figure was scanned, scaled up 3.5 times in 3D 
software and milled in $2,80 \mathrm{~m}$ height from a massive block of 1.7 tons limestone.
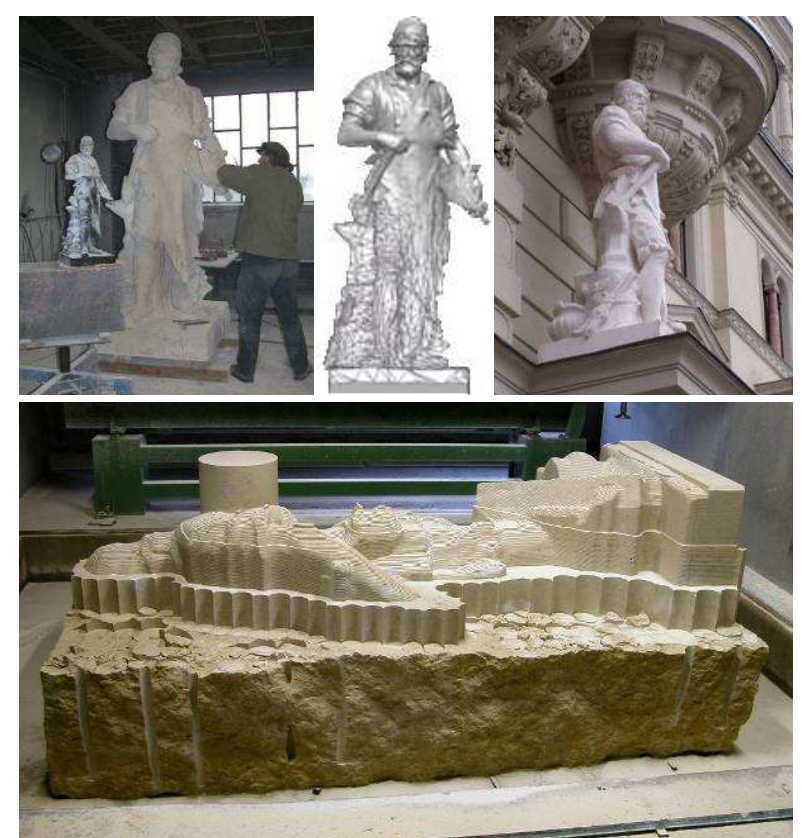

Figure 11: model of the allegory statue "craft" and it's 3,5 times scaled copy derived from scanning and CNCmilling, large copy $(2,8 \mathrm{~m})$ on the town hall in Graz, Austria

\subsection{Conservation Aid}

The use of 3D scan data and CNC-milling is also applied as restoration and conservation aid, i.e. to stabilize sculptures and other objects. This process also benefits strongly from the technical progress.

In 2004 a panel painting $(1.2 \times 1,8 \mathrm{~m})$ which was damaged strongly by insects, was stabilized with an Aramid-honeycomb panel (Hamann 2004). To adapt the honeycomb panel to the uneven surface of the deteriorated backside of the painting, 3D scan data and CNC-milling was used. The damaged backside of the panel was scanned and the data mirrored in 3D software.
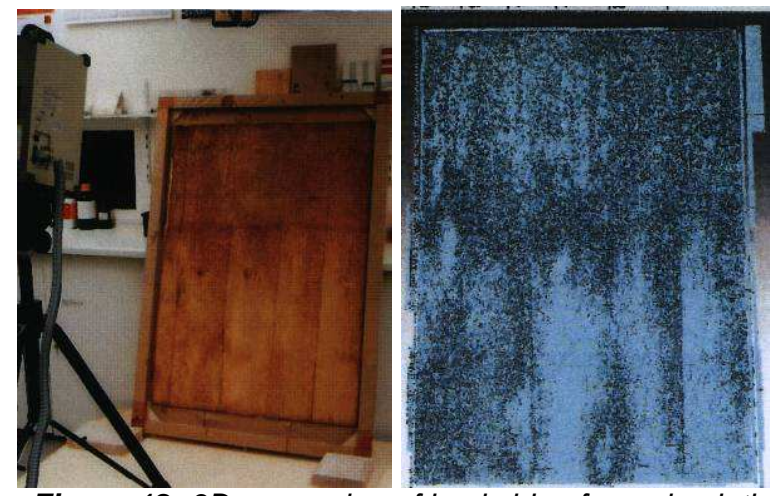

Figure 12: 3D measuring of backside of panel painting, $3 D$ scan data
The mirrored data were input to a CNC-machine which was milling the counter part to the damaged surface.
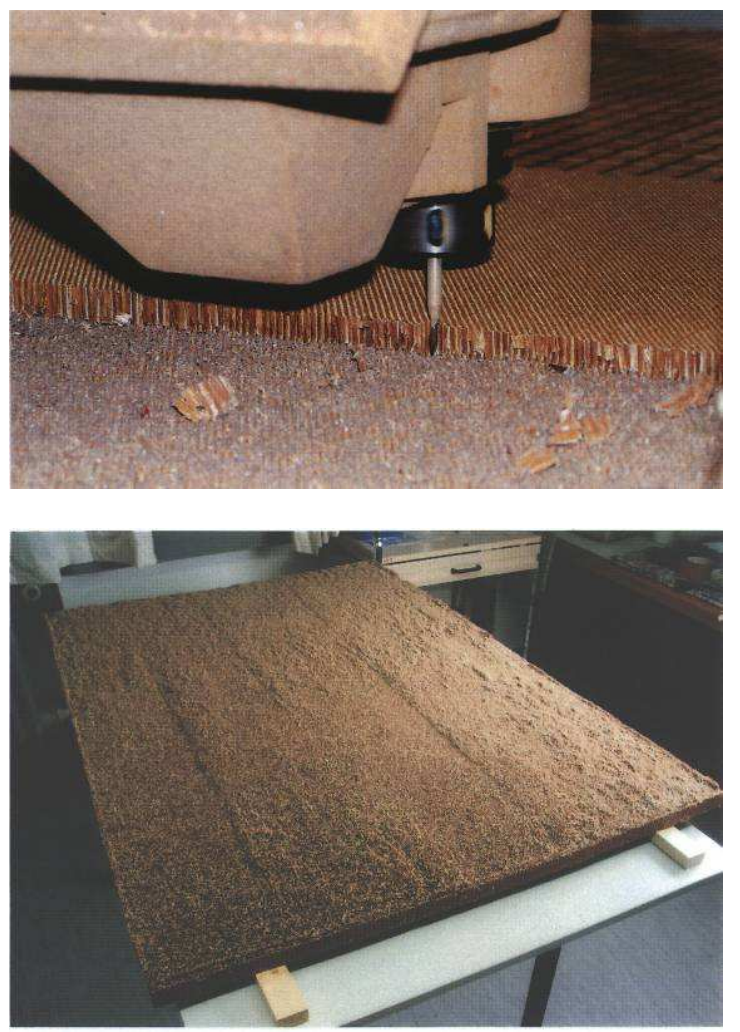

Figure 13: Milling process of the honeycomb panel, milled counterpart surface to stabilize the painting

The milled honeycombed panel with the counter part of the damaged backside of the panel painting was then glued to the panel painting for stabilization. Thus, no original substance for plaining the surface was lost.
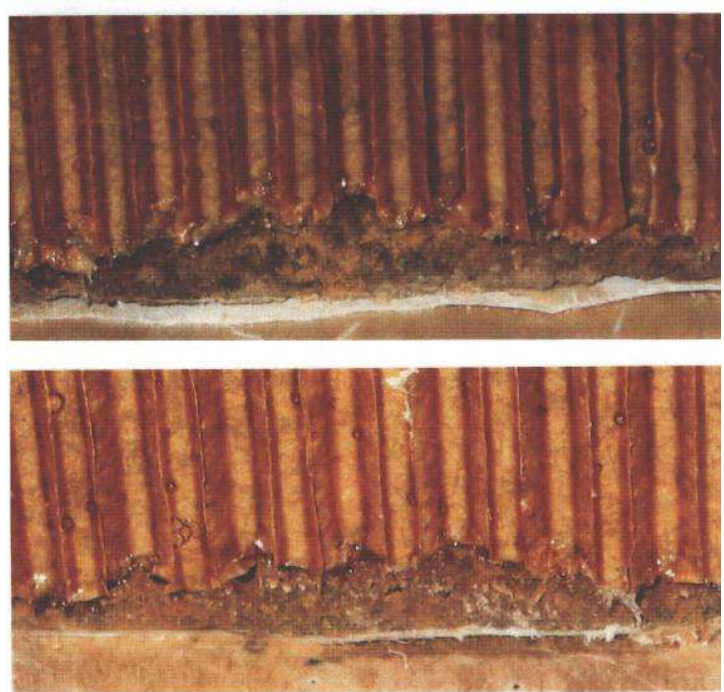

Figure 14: Honeycomb panel and panel painting glued together 


\subsection{Transportation Packaging}

Scan data can also be used to produce optimal transportation packaging. The scan data of an object can be proceeded in 3D software packages to calculate the negative shape, remove undercuts and to split the shape into blocks for easy packaging.
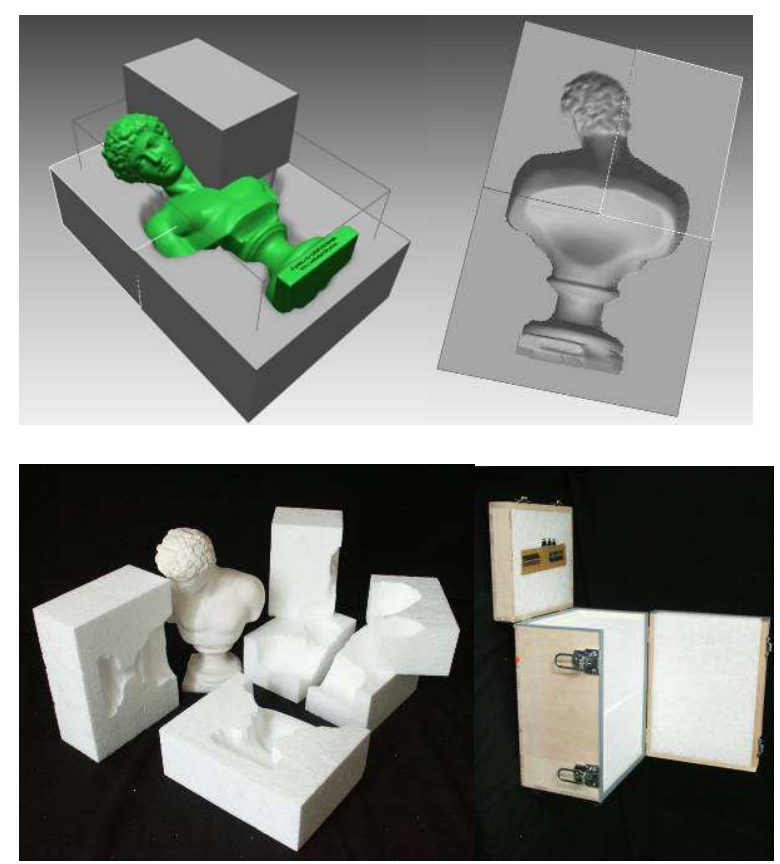

Figure 15: Calculation of negative shape in 3D software, transportation packaging milled from foam

Any material can be milled; also an offset can be calculated to insert special materials if necessary. Thus fragile objects can be safely transported.

\subsection{Museum Shops}

For multiple and cheaper copies of an object, e.g. for museum shops, a mould can be produced from $3 \mathrm{D}$ scan data. Thus an object can be reproduced multiple times in different sizes and materials. Direct moulding which may cause damage to the object can be avoided.
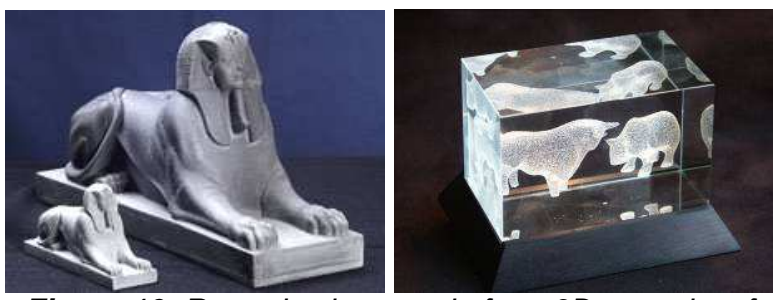

Figure 16: Reproductions made from 3D scan data for museum shops

\section{CONCLUSION}

The use of close range shape and dimension measurement systems in museums, archaeological sites as well as restoration and conservation of historic objects has been greatly improved within the past decade.

At the beginning of the year 2000 the first trials were undertaken to test the ability of $3 \mathrm{D}$ scanning and related processes such as CNC milling, etc. for Cultural Heritage. In 2004, larger projects were already completed successfully, e.g. copying of 2.8 $\mathrm{m}$ high stone statue.

Today the techniques of non-invasive 3D scanning are widely known and more and more common within Cultural Heritage for digital archiving, documentation, copying and reconstruction, conservation aid, etc.

\section{REFERENCES}

Adolf, S. (2001) Optical techniques for multidimensional measurement of art objects Deformation measurement and digital evaluation of panel paintings. Diploma thesis, FH Cologne, 2001

Köhler, S. (2000), Möglichkeiten zur Herstellung aufsteckbarer Ergänzungen für Holzskulpturen. Diploma thesis, FH Cologne, 2000

Mühlbauer, A. (2004), 3D Verfahren in der Denkmalpflege. Der Denkmalbrief, 8, March 2004. www.formscan.de

Hamann, I. (2004), Stabilisierung eines gedünnten Holztafelbildes. Versuch der praktischen Umsetzung mit einer Aramid-Wabenplatte an einem Epitaph von 1585. Diploma thesis, FH Hildesheim, 2004 